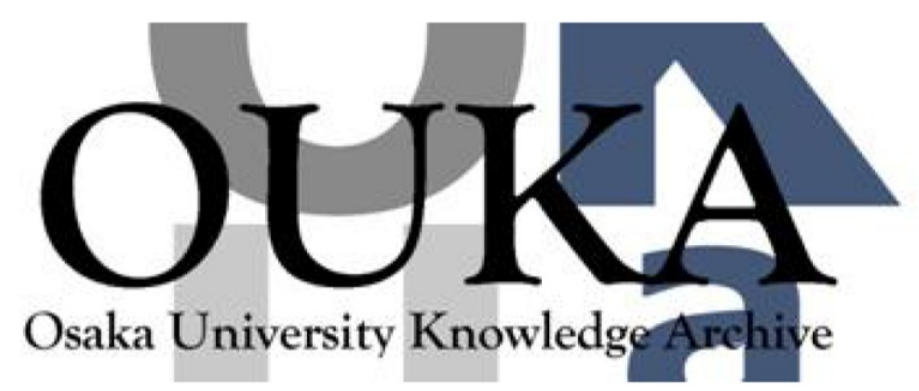

\begin{tabular}{|c|l|}
\hline Title & $\begin{array}{l}\text { Laser action in polydialkylfluorene fi lms : } \\
\text { Influence of low-temperature thermal treatment }\end{array}$ \\
\hline Author(s) & $\begin{array}{l}\text { Shkunov, M. N.; Österbacka, R. ; Fuji i, A. et } \\
\text { al. }\end{array}$ \\
\hline Citation & Applied Physics Letters. 74(12) p. 1648-p. 1650 \\
\hline Issue Date & $1999-03-18$ \\
\hline oaire:version & VoR \\
\hline URL & https://hdl. handle. net/11094/75652 \\
\hline rights & \\
\hline Note & \\
\hline
\end{tabular}

Osaka University Knowledge Archive : OUKA

https://ir. Library. osaka-u. ac. jp/

Osaka University 


\section{Laser action in polydialkylfluorene films: Influence of low-temperature thermal treatment}

Cite as: Appl. Phys. Lett. 74, 1648 (1999); https://doi.org/10.1063/1.123642

Submitted: 16 December 1998 . Accepted: 25 January 1999 . Published Online: 18 March 1999

M. N. Shkunov, R. Österbacka, A. Fujii, K. Yoshino, and Z. V. Vardeny

ARTICLES YOU MAY BE INTERESTED IN

Light amplification and gain in polyfluorene waveguides

Applied Physics Letters 81, 415 (2002); https://doi.org/10.1063/1.1494473

Amplified spontaneous emission and efficient tunable laser emission from a substituted thiophene-based oligomer

Applied Physics Letters 81, 3534 (2002); https://doi.org/10.1063/1.1519735

Influence of the orientation of liquid crystalline poly(9,9-dioctylfluorene) on its lasing properties in a planar microcavity

Applied Physics Letters 80, 4088 (2002); https://doi.org/10.1063/1.1481977

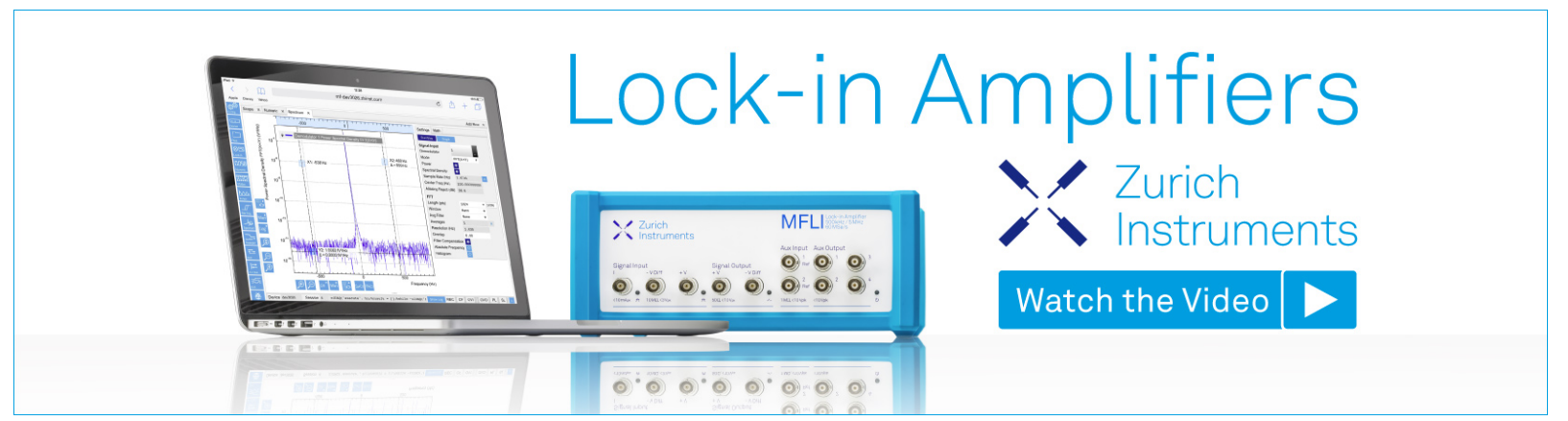




\title{
Laser action in polydialkylfluorene films: Influence of low-temperature thermal treatment
}

M. N. Shkunov

Department of Physics, University of Utah, Salt Lake City, Utah 84112-0830

R. Österbacka ${ }^{a)}$

Department of Physics, University of Utah, Salt Lake City, Utah 84112-0830

and Department of Physics, Abo Akademi University, Porhansgatan 3, Fin-20500 Turku, Finland

\author{
A. Fujii and K. Yoshino \\ Department of Electronic Engineering, Osaka University, Suita, Osaka 565-0871, Japan
}

Z. V. Vardeny

Department of Physics, University of Utah, Salt Lake City, Utah 84112-0830

(Received 16 December 1998; accepted for publication 25 January 1999)

\begin{abstract}
We have used a variety of optical probes to investigate the changes occurring upon low-temperature thermal treatment to the emissive properties of dialkyl substituted polyfluorene thin films. We found that the low-temperature-driven morphological changes involving aggregates formation, which are observed in the absorption and photoluminescence spectra at low excitation intensities, give rise at high excitation intensities to laser action in the blue spectral range. In some cases, switching between two stimulated emission bands was also observed. (c) 1999 American Institute of Physics.
\end{abstract} [S0003-6951(99)02412-2]

The recent demonstration of emission spectral narrowing (SN) in thin solid films of several $\pi$-conjugated polymers ${ }^{1-3}$ has generated wide-spread interest in the phenomenon of laser action in these materials. It is now generally believed that $\mathrm{SN}$ in neat polymer films is caused by amplified spontaneous emission (ASE) enhanced by optical waveguiding in the polymer films. ${ }^{4} \mathrm{SN}$ occurs at the moderately high exciton density of $10^{17}-10^{19} \mathrm{~cm}^{-3}$, at wavelengths where the stimulated emission (SE) overcomes self-absorption and is accompanied by substantial lifetime shortening. ${ }^{3}$ Extensive studies of various lasing cavities using these polymers as active materials have been directed towards the realization of electrically driven organic solid-state lasers. ${ }^{1,5-8}$ Yet, the SN mechanism itself is not very well understood. For example, the reason why some conjugated polymers which have a high photoluminescence quantum yield nevertheless lack SN is not exactly known. Also, the influence of morphological changes involving aggregates formation in the polymer film on its laser action at high excitation intensities has not, as yet, been thoroughly studied.

In this work, we investigate the optical emission properties of dialkyl-substituted polyfluorene (PDAF) thin films, which undergo morphological changes upon lowtemperature thermal treatment, ${ }^{9}$ using both low and high excitation intensities. In particular, we studied films of di-octyl substitution, PDAF8, the backbone of which is shown in Fig. 1(a), inset. We note that the side groups responsible for the polymer solubility are well separated from the side groups on the adjacent fluorene units and this dramatically reduces the steric interaction that twists the conjugated repeat units from the planar configuration. In addition, PDAF films can be controllably modified without changes in the polymer chemical

${ }^{a)}$ Also at the Graduate School of Materials Research, Turku, Finland. structure. For example, PDAF melts into a liquid-crystalline phase at $\approx 170{ }^{\circ} \mathrm{C}$ and can be quenched as a glass or crystallites by either fast or slow cooling from the melt. ${ }^{10}$ Moreover, this polymer is a blue emitter, which has recently attracted much interest for use in electroluminescence
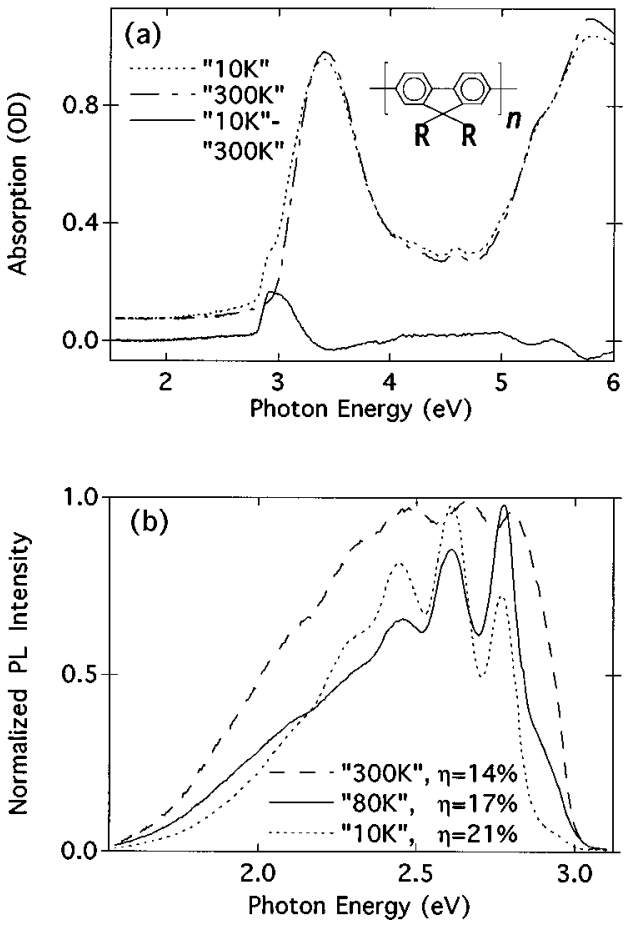

FIG. 1. Changes in the absorption (a) and photoluminescence (b) spectra of PDAF8 films upon low-temperature thermal treatment, as measured at room temperature. The " $10 \mathrm{~K}$ " and " $80 \mathrm{~K}$ " films were cooled to 10 and $80 \mathrm{~K}$, respectively, whereas the " $300 \mathrm{~K}$ " film was never cooled below room temperature. The inset in (a) shows the polymer repeat unit, whereas the inset in (b) gives the respective films PL quantum efficiency $\eta$. 
devices ${ }^{11}$ and light-emitting electrochemical cells. ${ }^{12}$ Preliminary studies have shown that dramatic changes occur in the optical spectra of PDAF films upon low-temperature thermal treatment. ${ }^{9}$ In addition, using PDAF as the active medium, lasing in solutions, and ASE in thin films were also observed. ${ }^{13,14}$ However, the influence of the changes induced by the low-temperature thermal treatment on the laser-action properties, has not been investigated to the best of our knowledge.

We have measured the changes induced upon lowtemperature thermal treatment in the absorption $(\alpha)$ and photoluminescence (PL) spectra at low excitation intensities, and $\mathrm{SN}$ at high excitation intensities. The $\alpha(\omega)$ spectrum was measured using a Perkin-Elmer Lambda 9 UV-Vis spectrometer. The PL was measured using a 1/4 met Jarell-Ash spectrometer, where the excitation beam was from an $\mathrm{Ar}^{+}$ laser operating at $363 \mathrm{~nm}$. All measurements were done at room temperature. We measured the PL quantum yield, $\eta_{\mathrm{PL}}$ per absorbed photon by an integrating sphere with $363 \mathrm{~nm}$ excitation.

For the laser-action measurements at high excitation intensities, we used a laser beam with 100 ps pulses at $100 \mathrm{~Hz}$ repetition rate with the energy per pulse ranging from 0.1 to $50 \mu \mathrm{J}$, provided by a $\mathrm{Nd}$ :YAG regenerative laser amplifier, which was frequency tripled at $355 \mathrm{~nm}$. The pump beam was focused onto a circular illuminated area of about $1 \mathrm{~mm}^{2}$ on the PDAF8 film. The emission was collected by a lens and analyzed using a 0.6 met spectrometer and a charge-coupled device camera with $\sim 3 \AA$ spectral resolution.

The PDAF8 was synthesized by a method published in Ref. 15 using a Suzuki coupling reaction..$^{16}$ Thin films for the spectroscopic studies were prepared by spin coating from solution of THF, which is known to be a good solvent for PDAF polymers. ${ }^{9}$ Three initially identical films were prepared, where two of them were subject to low-temperature thermal treatment. The three films are dubbed here as follows: the "300 K" sample was kept at room temperature; the " $80 \mathrm{~K}$ " sample was cooled down to $80 \mathrm{~K}$ in a cold finger cryostat followed by a slow warm-up to room temperature; and the " $10 \mathrm{~K}$ " sample was heat treated similar to the " 80 K" films, but at helium atmosphere at a temperature of $10 \mathrm{~K}$. The optical changes in the films reported here are all irreversible on a time scale of several months, but can be eliminated by heating the samples up to the glass transition temperature at $\sim 170{ }^{\circ} \mathrm{C}$.

In Fig. 1(a) we show the room-temperature absorption spectra of the " $300 \mathrm{~K}$ " and " $10 \mathrm{~K}$ " films; similar results were obtained with the " $80 \mathrm{~K}$ " film. It is seen that the lowtemperature thermal treatment enhances an absorption band at energies below the initial PDAF8 optical gap. ${ }^{9}$ This band is better seen in the absorption difference spectrum, $\Delta \alpha$ $=\alpha($ " $10 \mathrm{~K}$ ") $)-\alpha($ " $300 \mathrm{~K}$ ") $)$; the new band peaks at $\sim 3$ $\mathrm{eV}$ with an onset at $\sim 2.88 \mathrm{eV}$. From the $\Delta \alpha$ spectrum we conclude that a fraction of the material, estimated at $\sim 8 \%$, has been transformed by the thermal treatment into a new morphology; for the " $80 \mathrm{~K}$ " sample this fraction is less than $1 \%$. The nature of the new molecular structure is not exactly known, but from the redshifted absorption spectrum we speculate that it corresponds to a well-defined state of superior order with enhanced conjugation. Such an order state may be formed, for example, by chain aggregation where the polymer adopts a more planar conformation. We note similar aggregation discovered in other polymer films made from the dialkoxy derivatives of poly ( $p$-phenylene vinylene) and polythiophene. ${ }^{17,18}$

The corresponding changes occurring in the PL spectra upon the thermal treatment are much clearer, as shown in Fig. 1(b). All three PL spectra are structured at room temperature with up to five phonon replica. However, the PL spectra of the " $80 \mathrm{~K}$ " and " $10 \mathrm{~K}$ " films show much more pronounced phonon replica. In addition, the PL spectra of these two films also show a redshift of order $30 \mathrm{meV}$, in agreement with the redshift seen in the $\Delta \alpha$ spectrum [Fig. $1(\mathrm{a})]$. We note that the $0-0$ PL band is weaker in the " 10 $\mathrm{K}$ " film compared to the " $80 \mathrm{~K}$ " and " $300 \mathrm{~K}$ " films, and this is caused by the enhanced self-absorption due to the redshifted absorption in this film [Fig. 1(a)]. The PL quantum yields also follow the spectroscopic changes in PL observed upon thermal treatment, with $\eta_{\mathrm{PL}}$ (" $10 \mathrm{~K}$ ') $>\eta_{\mathrm{PL}}\left(\right.$ " $80 \mathrm{~K}$ ') $>\eta_{\mathrm{PL}}$ (" $300 \mathrm{~K}$ '). We note that $\eta_{\mathrm{PL}}$ reaches $21 \%$ for the " $10 \mathrm{~K}$ " sample in agreement with the superior order of this film.

The laser-action properties of the three PDAF8 films at high excitation intensities $I$ are shown in Fig. 2. The " 300 K" film [Fig. 2(a)] does not show SN at all $I$. The two other films, however, show SN at 442 and $463 \mathrm{~nm}$, respectively, where the broad PL band of $120 \mathrm{~nm}$ collapses at $I>I_{0}$ into a narrow band of $4 \mathrm{~nm}$ spectral width. It is notable that the pristine PDAF8 film does not show SN, but aggregates formation in the heat-treated film results in SN. We speculate that this is due to the superior order in the film that is generated upon thermal treatment. The excitation intensity threshold $I_{0}$ for SN is $7.4 \mu \mathrm{J}$ for the " $80 \mathrm{~K}$ " film and $1.5 \mu \mathrm{J}$ for the " $10 \mathrm{~K}$ " film. That $I_{0}$ is lower for the " $10 \mathrm{~K}$ " film is in agreement with its higher PL quantum yield [Fig. 1(b)].

The most interesting behavior at high $I$, however, is the SN switching in the " $80 \mathrm{~K}$ " film from a SE band centered at $463 \mathrm{~nm}$ to another SE band at $442 \mathrm{~nm}$, which occurs at $I_{1}$ $=11 \mu \mathrm{J}>I_{0}$; the " $10 \mathrm{~K}$ " film, on the other hand, shows only a single SE band at $463 \mathrm{~nm}$. The SN switching between the two wavelengths in the " $80 \mathrm{~K}$ " film may be explained by an interplay between optical gain, coupled with an optical absorption saturation at $I_{1}$ as follows. At moderately high $I$, the effective optical gain $g_{\text {eff }}$ in the polymer film follows the relation: ${ }^{19}$

$$
g_{\text {eff }}(\omega)=N \sigma(\omega)-\alpha(\omega),
$$

where $N(\sim I)$ is the exciton density, $\sigma(\omega)$ is their optical gain cross-section spectrum that is mainly proportional to the PL spectrum, and $\alpha(\omega)$ is the absorption loss in the tail. SE occurs at $I_{0}$ and $\omega_{0}$ such that $g_{\text {eff }}\left(I_{0}, \omega_{0}\right)>0$. Since $N \sigma(\omega)$ in Eq. (1) increases linearly with $I$, then SE switching from one $\omega_{0}$ to the other must be driven by nonlinear changes in $g_{\text {eff }}(\omega)$, and thus, can only occur if some changes in $\alpha(\omega)$ happen at high $I$. This may occur, for example, if $\alpha(\omega)$ in the tail is saturated or photobleached at high $I$ due to exciton energy migration into lower-energy states and, in particular, into the aggregate states. ${ }^{20} \mathrm{We}$ note that the aggregate absorption band in the " $80 \mathrm{~K}$ " film is quite small (smaller than $1 \%$ of the total $\alpha$ ), and therefore, this film is more suscep- 


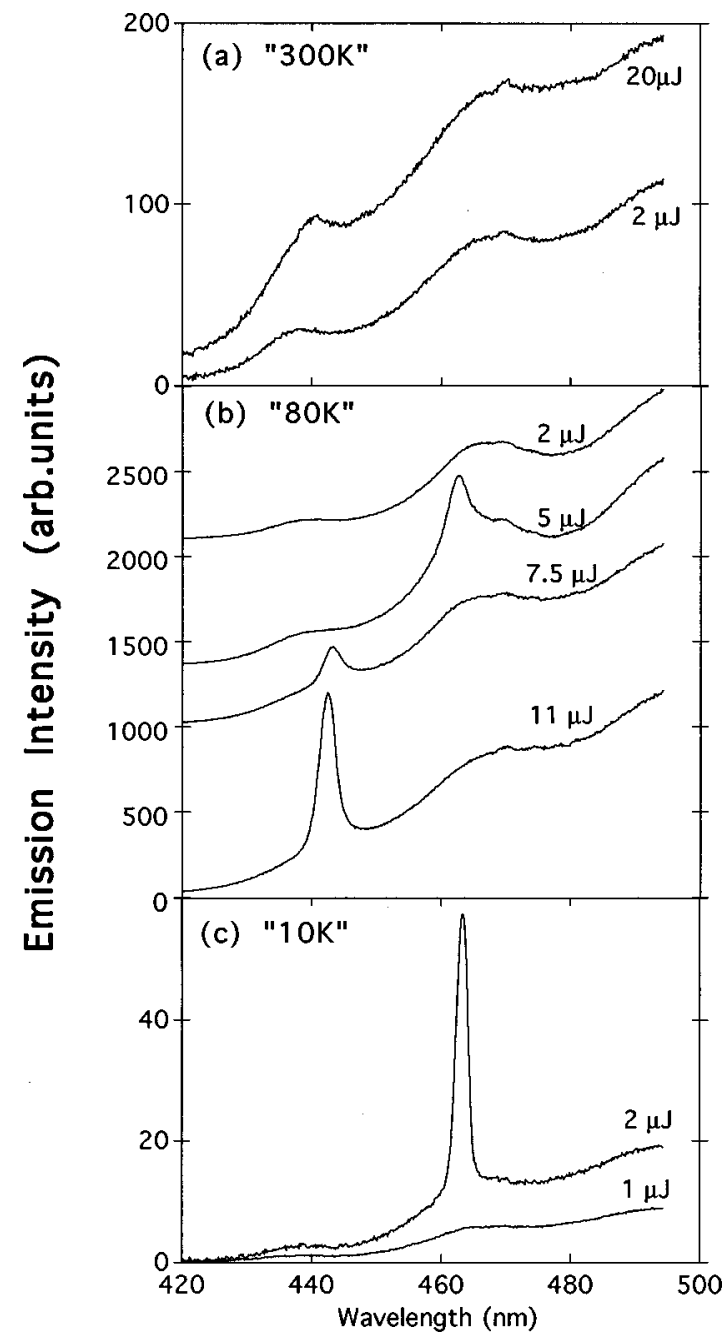

FIG. 2. The uncorrected emission spectra of (a) " $300 \mathrm{~K}$," (b) " $80 \mathrm{~K}$," and (c) " $10 \mathrm{~K}$ " PDAF8 films at high laser excitation intensities of $100 \mathrm{ps}$ at 355 $\mathrm{nm}$. The excitation intensity is given in energy per pulse, where the illuminated area on the films was $1 \mathrm{~mm}^{2}$. Narrow SE bands are formed in (b) and (c) at excitation intensity thresholds of 7.4 and $1.5 \mu \mathrm{J}$, respectively.

tible to optical saturation at high $I$, compared to that of the " $10 \mathrm{~K}$ " film where the aggregate absorption band is of the order of $8 \%$ [Fig. 1(a)].

From the PL spectrum at low $I$ [Fig. 1(b)], it is seen that in the " $80 \mathrm{~K}$ " film the $0-0$ emission band $(\sim 442 \mathrm{~nm})$ is stronger than that of $0-1$ band at $\sim 463 \mathrm{~nm}$; this is carried over to $\sigma(\omega)$ of this film. Nevertheless, $g_{\text {eff }}>0$ first occurs at the $0-1$ emission band rather than at the $0-0$ band because of $\alpha(\omega)$ [Eq. (1)], which is larger at $442 \mathrm{~nm}$ [Fig. 1(a)]. However, at sufficiently high $I, \alpha(442 \mathrm{~nm})$ is partially photobleached, resulting in $g_{\text {eff }}(442 \mathrm{~nm})>g_{\text {eff }}(463 \mathrm{~nm})$ and this causes SE switching between these two wavelengths. We also note from Fig. 1(b) that the $0-0$ emission band in the
" $10 \mathrm{~K}$ " film is much weaker than the $0-1$ emission band, and this is caused by the higher $\alpha$ in the tail for this sample [Fig. 1(a)]. Under these conditions $g_{\text {eff }}>0$ occurs first at the $0-1$ not at the $0-0$ band, and since photobleaching of $\alpha$ in the tail does not occur in this film because $\alpha$ is too high, then no SE switching is observed here.

The SE switching phenomenon from one band to the other at a certain threshold excitation intensity is a very interesting effect with potential applications ranging from optical switches, to saturable and inverse saturable absorbers. We note that the SE switching effect is unique for $\pi$-conjugated polymers, and stems from the pronounced phonon replica in the PL spectra of these materials.

This work was partially financed by the NSF, DMR 9732820. One of the authors (R.Ö.) acknowledges funding from the Swedish Academy of Engineering Sciences in Finland and the Neste, Ltd. Foundation.

${ }^{1}$ N. Tessler, G. J. Denton, and R. Friend, Nature (London) 382, 695 (1996). ${ }^{2}$ F. Hide, M. A. Diaz-Garcia, B. J. Schwartz, M. R. Anderson, Q. Pei, and A. J. Heeger, Science 273, 1833 (1996); F. Hide, B. J. Schwartz, M. A. Diaz-Garcia, and A. J. Heeger, Chem. Phys. Lett. 256, 424 (1996).

${ }^{3}$ S. V. Frolov, M. Ozaki, W. Gellerman, K. Yoshino, and Z. V. Vardeny, Jpn. J. Appl. Phys., Part 2 35, L1371 (1996); Phys. Rev. Lett. 78, 729 (1997).

${ }^{4}$ G. J. Denton, N. Tessler, M. A. Stevens, and R. M. Friend, Adv. Mater. 9, 547 (1997).

${ }^{5}$ S. V. Frolov, M. Shkunov, Z. V. Vardeny, and K. Yoshino, Phys. Rev. B 56, R4363 (1997); S. V. Frolov, A. Fujii, D. Chinn, Z. V. Vardeny, K. Yoshino, and R. V. Gregory, Appl. Phys. Lett. 72, 2811 (1998).

${ }^{6}$ V. G. Kozlov, V. Bulovic, P. E. Burrows, and S. R. Forrest, Nature (London) 389, 362 (1997).

${ }^{7}$ M. Berggren, A. Dodabalapur, R. E. Slusher, and Z. Bao, Appl. Phys. Lett. 71, 2230 (1997); Adv. Mater. 9, 968 (1997).

${ }^{8}$ M. D. McGehee, M. A. Diaz-Garcia, F. Hide, R. Gupta, E. K. Miller, D. Moses, and A. J. Heeger, Appl. Phys. Lett. 72, 1536 (1998).

${ }^{9}$ D. D. C. Bradley, M. Grell, X. Long, H. Mellor, A. Grice, M. Inbasekaran, and E. P. Woo, Proc. SPIE 3145, 254 (1997).

${ }^{10}$ M. Grell, X. Long, D. D. C. Bradley, M. Inbasekaran, and E. P. Woo, Adv. Mater. 9, 798 (1997).

${ }^{11}$ Y. Ohmori, M. Uchida, C. Morishima, A. Fujii, and K. Yoshino, Jpn. J. Appl. Phys., Part 2 32, L1663 (1993).

${ }^{12}$ Q. Pei and Y. Yang, J. Am. Chem. Soc. 118, 7416 (1996).

${ }^{13}$ X. Long, A. Malinowski, D. D. C. Bradley, M. Inbasekaran, and E. P. Woo, Chem. Phys. Lett. 272, 6 (1997).

${ }^{14}$ X. Long, M. Grell, A. Malinowski, D. D. C. Bradley, M. Inbasekaran, and E. P. Woo, Opt. Mater. 9, 70 (1998).

${ }^{15}$ Y. Ohmori, M. Uchida, K. Muro, and K. Yoshino, Jpn. J. Appl. Phys., Part 2 30, L1941 (1991)

${ }^{16}$ N. Miyaura and A. Suzuki, Chem. Rev. 95, 2457 (1990).

${ }^{17}$ B. M. W. Langeved-Voss, E. Peeters, R. A. Janssen, and E. W. Meijer, Synth. Met. 84, 611 (1997).

${ }^{18}$ J. W. Blatchford, T. L. Gustafson, A. J. Epstein, D. A. Vanden Bout, J. Kerimo, D. A. Higgins, P. F. Barbara, D. K. Fu, T. M. Swager, and A. G. MacDiarmid, Phys. Rev. B 54, R3683 (1996).

${ }^{19}$ S. V. Frolov, Z. V. Vardeny, and K. Yoshino, Phys. Rev. B 57, 9141 (1998).

${ }^{20}$ R. Kersting, U. Lemmer, R. F. Mahrt, K. Leo, H. Kurz, H. Bässler, and E. O. Göbel, Phys. Rev. Lett. 70, 3820 (1993). 